\title{
ANÁLISIS PANBIOGEOGRÁFICO DE LAS ESPECIES MEXICANAS DE PSELLIOPUS BERGROTH (HEMIPTERA: HETEROPTERA: REDUVIIDAE: HARPACTORINAE)
}

\author{
Ricardo Mariño-Pérez ${ }^{1}$, Harry Brailovsky ${ }^{1}$ y Juan J. Morrone ${ }^{2}$ \\ ${ }^{1}$ Departamento de Zoología, Instituto de Biología, UNAM, Departamento de Zoología, \\ Apdo. Postal 70153, 04510 México D. F. MÉXICO \\ ${ }^{2}$ Museo de Zoología "Alfonso L. Herrera”, Departamento de Biología Evolutiva, \\ Facultad de Ciencias, UNAM, Apdo. Postal 70-399, 04510 México D. F., MÉXICO
}

\begin{abstract}
RESUMEN
Se llevó a cabo un análisis panbiogeográfico de las especies mexicanas de Pselliopus Bergroth. El género contiene 27 especies, que se distribuyen desde Canadá hasta la Argentina. México es el país con el mayor número de especies (21), seguido de los Estados Unidos de América (6) y Guatemala, El Salvador, Honduras y Brasil (2). Se obtuvo un trazo generalizado que abarca la Sierra Madre Occidental, la Sierra Madre Oriental, la Costa del Pacífico Mexicano, el Golfo de México, la Cuenca del Balsas, el Eje Volcánico Transmexicano, la Sierra Madre del Sur y Chiapas. Se concluye que las especies de Pselliopus se distribuyen principalmente en la Zona de Transición Mexicana (ZTM) y la región Neotropical, y pertenecen al elemento Mesoamericano Tropical.

Palabras Clave: Heteroptera, Reduviidae, Pselliopus, Biogeografía, Zona de Transición Mexicana, región Neotropical, elemento Mesoamericano Tropical, México.
\end{abstract}

\begin{abstract}
A panbiogeographic analysis was carried out to analyze the geographical distribution of the Mexican species of Pselliopus Bergroth. The genus contains 27 species, ranged from Canada to Argentina. Mexico is the country with the highest number of species (21), followed by the United States of America (6), and Guatemala, El Salvador, Honduras and Brazil (2). One generalized tracks was recognized, comprising the Sierra Madre Occidental, the Sierra Madre Oriental, Mexican Pacific Coast, Mexican Gulf, Balsas Basin, Transmexican Volcanic Belt, Sierra Madre del Sur, and Chiapas. It is concluded that the species of Pselliopus are distributed mainly in the Mexican Transition Zone (MTZ) and the Neotropcial region, and belong to the Tropical Mesoamerican element.

Key Words: Heteroptera, Reduviidae, Pselliopus, Biogeography, Mexican Transition Zone, Neotropical region, Tropical Mesoamerican element, Mexico.
\end{abstract}

\section{INTRODUCCIÓN}

Las especies asignadas al género Pselliopus Bergroth depredan insectos y otros artrópodos terrestres, distribuyéndose desde Canadá hasta América del Sur. De acuerdo 
con Brailovsky y Barrera (2004), Pselliopus estaba integrado por 17 especies, diez de ellas mexicanas: P. inermis (Champion), P. latispina Hussey, P. lineaticeps (Champion), P. mexicanus (Champion), P. nigropictus (Champion), P. punctipes (Amyot y Serville), P. rufofasciatus (Champion), P. spinicollis (Champion), P. tuberculatus (Champion) y P. zebra (Stål). La revisión sistemática de Mariño-Pérez (2006) permitió elevar el número de especies mexicanas a 21, para un total de 27 especies americanas (Brailovsky et al., 2007). La información sobre la distribución de estas especies es de interés para llevar a cabo estudios biogeográficos de México.

Nuestro objetivo es llevar a cabo un análisis panbiogeográfico de las especies mexicanas de Pselliopus, con la finalidad de conocer si sus patrones de distribución coinciden con patrones de distribución conocidos para otros organismos y contribuir al planteamiento de hipótesis biogeográficas que expliquen la distribución espacial de este grupo.

\section{MATERIAL Y MÉTODOS}

Los datos de distribución se obtuvieron de Brailovsky et al. (2007). Se analizaron 20 (de un total de 21) especies mexicanas de Pselliopus, ya que P. rayonensis Brailovsky et al., se conoce de una sola localidad y por ello fue excluida del análisis. Si bien algunos de los trazos individuales atraviesan el Altiplano Mexicano, en realidad los registros son periféricos a esta provincia biogeográfica, por lo que la misma no fue incluida en el análisis.

El enfoque panbiogeográfico (Croizat 1958, 1964) destaca la importancia de la dimensión geográfica de la biodiversidad para permitir una comprensión más adecuada de los patrones y procesos evolutivos. El método panbiogeográfico (Morrone \& Crisci, 1995; Craw et al. 1999; Morrone, 2004) consiste en marcar en mapas las localidades de distribución de diferentes taxones y luego unirlas mediante líneas de menor distancia, denominados trazos individuales, los cuales pueden ser orientados con base en los criterios de centro de masa, información filogenética y línea de base. Cuando diferentes trazos individuales coinciden se considera que determinan un trazo generalizado, el cual permite sostener la existencia de una biota distribuida ampliamente en el pasado y fragmentada por eventos vicariantes. Si dos o más trazos generalizados confluyen en una misma área, ésta constituye un nodo, que posee una composición biótica múltiple, posiblemente en tiempos geológicos diferentes. Los trazos generalizados, líneas de base y nodos son marcados en un mapa que representa la hipótesis obtenida. En este análisis, los trazos individuales no fueron orientados, debido a que de los tres criterios que pueden usarse

para la orientación, el menos problemático sería el de la línea de base, pero cuando el análisis se realiza a escala continental, resulta difícil de aplicar (Morrone 2004). 
Con el objeto de obtener trazos generalizados, aplicamos el análisis de parsimonia de endemismos con eliminación progresiva de caracteres (PAE-PCE) (Rosen 1988; Morrone \& Crisci 1995; Luna-Vega et al. 2000). Comprende los siguientes pasos (Morrone 2004): (1) construir los trazos individuales, conectando las localidades de distribución mediante un árbol de tendido mínimo; (2) construir una matriz de áreas por trazos individuales, donde la presencia se representa con un " 1 " y la ausencia con un "0", y añadiendo un área "externa" con todos "0" para enraizar el cladograma; (3) analizar la matriz con un algoritmo de parsimonia y conectar las áreas incluidas en cada clado sustentado por al menos dos trazos individuales como parte de un mismo trazo generalizado; (4) desconectar los trazos individuales que sustentan los clados obtenidos y analizar nuevamente la matriz para buscar clados alternativos; y (5) indicar en un mapa los trazos generalizados y (si existen) los nodos.

\section{RESULTADOS}

En el cuadro 1 se resume la distribución de Pselliopus en América. Las especies se distribuyen principalmente en México (21 de un total de 27). La especie $P$. cinctus se distribuye en Canadá y los Estados Unidos, países donde se distribuyen también $P$. barberi, P. latifasciatus, P. marmorosus, P. spinicollis y P. zebra. Para América Central se han registrado especies en Guatemala, El Salvador, Honduras y Panamá; en todos estos países se ha citado $P$. zebra, además para el primero $P$. infuscatus, para el segundo $P$. punctipes y para el tercero $P$. karlenae. En América del Sur se ha registrado a $P$. ornaticeps para la Argentina y Brasil; de este último país también se conoce a $P$. limai (Maldonado-Capriles 1990). Es posible que existan más especies en las montañas de América Central y del Sur, por lo que se requieren muestreos en estas zonas. Por otra parte, P. zebra es la especie de distribución más amplia, habiéndosela registrado en seis países; sin embargo, tiene problemas taxonómicos y no se ha establecido claramente si se trata de una especie variable o las variaciones corresponden a especies diferentes, ya que no se analizaron suficientes ejemplares.

En las figuras 1-7 se presentan los trazos individuales de las especies mexicanas de Pselliopus y en el cuadro 2 se resume su distribución geográfica, de acuerdo con el esquema de Morrone (2005). Las provincias con menor número de especies son California, Baja California, Sonora y la Península de Yucatán, con una especie cada una. Para la provincia del Altiplano Mexicano, aunque existen registros de varias especies, estos corresponden siempre a los límites con provincias adyacentes, donde hay registros de dichas especies. Además, al no existir un solo registro alejado de las cadenas montañosas que rodean al Altiplano y a pesar de intensas colectas en la zona, se considera que Pselliopus está ausente de esta provincia. La provincia de Tamaulipas 
Mariño-Pérez et al.: Análisis panbiogeográfico de Pselliopus

\section{Cuadro 1}

Distribución de las especies de Pselliopus analizadas por países. Can: Canadá, EUA: Estados Unidos de América, Méx: México, Gua: Guatemala, Sal: El Salvador, Hon: Honduras, Pan: Panamá, Arg: Argentina y Bra: Brasil.

\begin{tabular}{|c|c|c|c|c|c|c|c|c|c|c|}
\hline Especies & Can & EUA & Méx & Gua & Sal & Hon & Pan & Arg & Bra & Total \\
\hline P. barberi & & $\mathrm{X}$ & & & & & & & & 1 \\
\hline P. cinctus & $\mathrm{X}$ & $\mathrm{X}$ & & & & & & & & 2 \\
\hline P. dantei & & & $X$ & & & & & & & 1 \\
\hline P. flaviceps & & & $\mathrm{X}$ & & & & & & & 1 \\
\hline P. inermis & & & $\mathrm{X}$ & & & & & & & 1 \\
\hline P. infuscatus & & & & $\mathrm{X}$ & & & & & & 1 \\
\hline P. ivanicus & & & $\mathrm{X}$ & & & & & & & 1 \\
\hline P. karlenae & & & $\mathrm{X}$ & & & $\mathrm{X}$ & & & & 2 \\
\hline P. latifasciatus & & $\mathrm{X}$ & & & & & & & & 1 \\
\hline P. latispina & & & $\mathrm{X}$ & & & & & & & 1 \\
\hline P. limai & & & & & & & & & $\mathrm{X}$ & 1 \\
\hline P. lineaticeps & & & $X$ & & & & & & & 1 \\
\hline P. majesticus & & & $\mathrm{X}$ & & & & & & & 1 \\
\hline P. mexicanus & & & $\mathrm{X}$ & & & & & & & 1 \\
\hline P. nigropictus & & & $\mathrm{X}$ & & & & & & & 1 \\
\hline P. ornaticeps & & & & & & & & $\mathrm{X}$ & $\mathrm{X}$ & 2 \\
\hline P. punctipes & & & $\mathrm{X}$ & & $\mathrm{X}$ & & & & & 2 \\
\hline P. rufofasciatus & & & $\mathrm{X}$ & & & & & & & 1 \\
\hline P. cosmopolites & & & $X$ & & & & & & & 1 \\
\hline P. mirabilis & & & $\mathrm{X}$ & & & & & & & 1 \\
\hline P. marmorosus & & $\mathrm{X}$ & $\mathrm{X}$ & & & & & & & 2 \\
\hline P. promeceops & & & $\mathrm{X}$ & & & & & & & 1 \\
\hline P spinicollis & & $\mathrm{X}$ & $\mathrm{X}$ & & & & & & & 2 \\
\hline P. tuberculatus & & & $\mathrm{X}$ & & & & & & & 1 \\
\hline P. ventus & & & $\mathrm{X}$ & & & & & & & 1 \\
\hline P. zebra & & $\mathrm{X}$ & $X$ & $\mathrm{X}$ & $\mathrm{X}$ & $\mathrm{X}$ & $\mathrm{X}$ & & & 6 \\
\hline Total & 1 & 6 & 21 & 2 & 2 & 2 & 1 & 1 & 2 & \\
\hline
\end{tabular}

presenta dos especies, las tres provincias del dominio Mesoamericano (Golfo de México, Chiapas y Costa Pacífica Mexicana) presentan cuatro, cinco y siete especies, respectivamente. Las provincias con mayor número de especies pertenecen a la Zona de Transición Mexicana (Halffter 1987): Sierra Madre Occidental (8 especies), Sierra Madre Oriental (8), Sierra Madre del Sur (9), Cuenca del Balsas (8) y Eje Volcánico Transmexicano (12). Es posible que existan más especies en la Sierra Madre Occidental, ya que tres especies nuevas tienen registros en ella y una especie nueva tiene su único registro a pocos kilómetros de la provincia. 


\section{Cuadro 2}

Distribución de las especies de Pselliopus de México, de acuerdo con la propuesta de regionalización de Morrone (2005).

\begin{tabular}{|c|c|c|c|}
\hline Regiones/ZTM & Dominios & Provincias & Especies \\
\hline \multirow[t]{4}{*}{ Neártica } & Neártico Californiano & California & P. spinicollis \\
\hline & & Baja California & P. spinicollis \\
\hline & Neártico Continental & Sonora & P. rayonensis \\
\hline & & Tamaulipas & P. latispina y $P$. cosmopolites \\
\hline \multirow[t]{5}{*}{$\begin{array}{l}\text { Zona de } \\
\text { Transición } \\
\text { Mexicana }\end{array}$} & Mexicano de Montaña & Sierra Madre Occidental & $\begin{array}{l}\text { P. inermis, } P \text {. ivanicus, } P \text {. lineaticeps, } P . \\
\text { punctipes, } P \text {. mirabilis, } P \text {. marmorosus, } P \text {. } \\
\text { promeceops y } P \text {. tuberculatus }\end{array}$ \\
\hline & & Sierra Madre Oriental & $\begin{array}{l}\text { P. inermis, } P \text {. latispina, } P \text {. nigropictu s, } P \text {. } \\
\text { punctipes, } P \text {. cosmopolites, } P \text {. tuberculatus, } \\
P \text {. ventus y } P \text {. zebra }\end{array}$ \\
\hline & & $\begin{array}{l}\text { Eje Volcánico } \\
\text { Transmexicano }\end{array}$ & $\begin{array}{l}\text { P. flaviceps, } P \text {. inermis, } P \text {. ivanicus, } P \text {. } \\
\text { mexicanus, } P \text {. nigropictus, } P \text {. punctipes, } P \text {. } \\
\text { rufofasciatus, } P \text {. cosmopolites, } P \text {. } \\
\text { promeceops, } P \text {. tuberculatus, } P \text {. ventus y } P \text {. } \\
\text { zebra }\end{array}$ \\
\hline & & Cuenca del Balsas & $\begin{array}{l}\text { P. flaviceps, } P \text {. inermis, } P \text {. ivanicus, } P \text {. } \\
\text { nigropictus, } P \text {. promeceops } P \text {. punctipes, } P \text {. } \\
\text { rufofasciatus y } P \text {. ventus }\end{array}$ \\
\hline & & Sierra Madre del Sur & $\begin{array}{l}\text { P. dantei, } P \text {. inermis, } P \text {. ivanicus, } P \text {. } \\
\text { majesticus, } P \text {. nigropictus, } P \text {. rufofasciatus, } \\
\text { P. cosmopolites, } P \text {. ventus y } P \text {. zebra }\end{array}$ \\
\hline \multirow[t]{4}{*}{ Neotropical } & Antillano & Península de Yucatán & P. punctipes \\
\hline & Mesoamericano & Costa Pacífica Mexicana & $\begin{array}{l}\text { P. flaviceps, } P \text {. lineaticeps, } P \text {. majesticus, } P \text {. } \\
\text { nigropictus, } P \text {. punctipes, } P \text {. promeceops } \mathrm{y} \\
\text { P. zebra }\end{array}$ \\
\hline & & Golfo de México & $\begin{array}{l}\text { P. flaviceps, P. nigropictus, P. punctipes y } \\
\text { P. zebra }\end{array}$ \\
\hline & & Chiapas & $\begin{array}{l}\text { P. inermis, } P \text {. karlenae, } P \text {. majesticus, } P \text {. } \\
\text { punctipes y } P \text {. ventus }\end{array}$ \\
\hline
\end{tabular}

Del análisis de la matriz de datos (Cuadro 3), se obtuvieron cuatro cladogramas de 36 pasos, índice de consistencia de 0.55 e índice de retención de 0.62 . A partir del único componente sustentado por dos especies ( $P$. nigropictus y $P$. zebra) en su consenso estricto (Fig. 8), se obtuvo un trazo generalizado que comprende básicamente la Sierra Madre Oriental, la Costa del Pacífico Mexicano, el Golfo de México, la Cuenca del Balsas, el Eje Volcánico Transmexicano y la Sierra Madre del Sur. Luego de eliminar los trazos individuales que lo sustentan, el análisis dio 12 cladogramas de 31 pasos, índice de consistencia de 0.58 e índice de retención de 0.62 ; en seis de estos cladogramas, se halló un trazo generalizado que comprende las mismas áreas que el anterior, además 

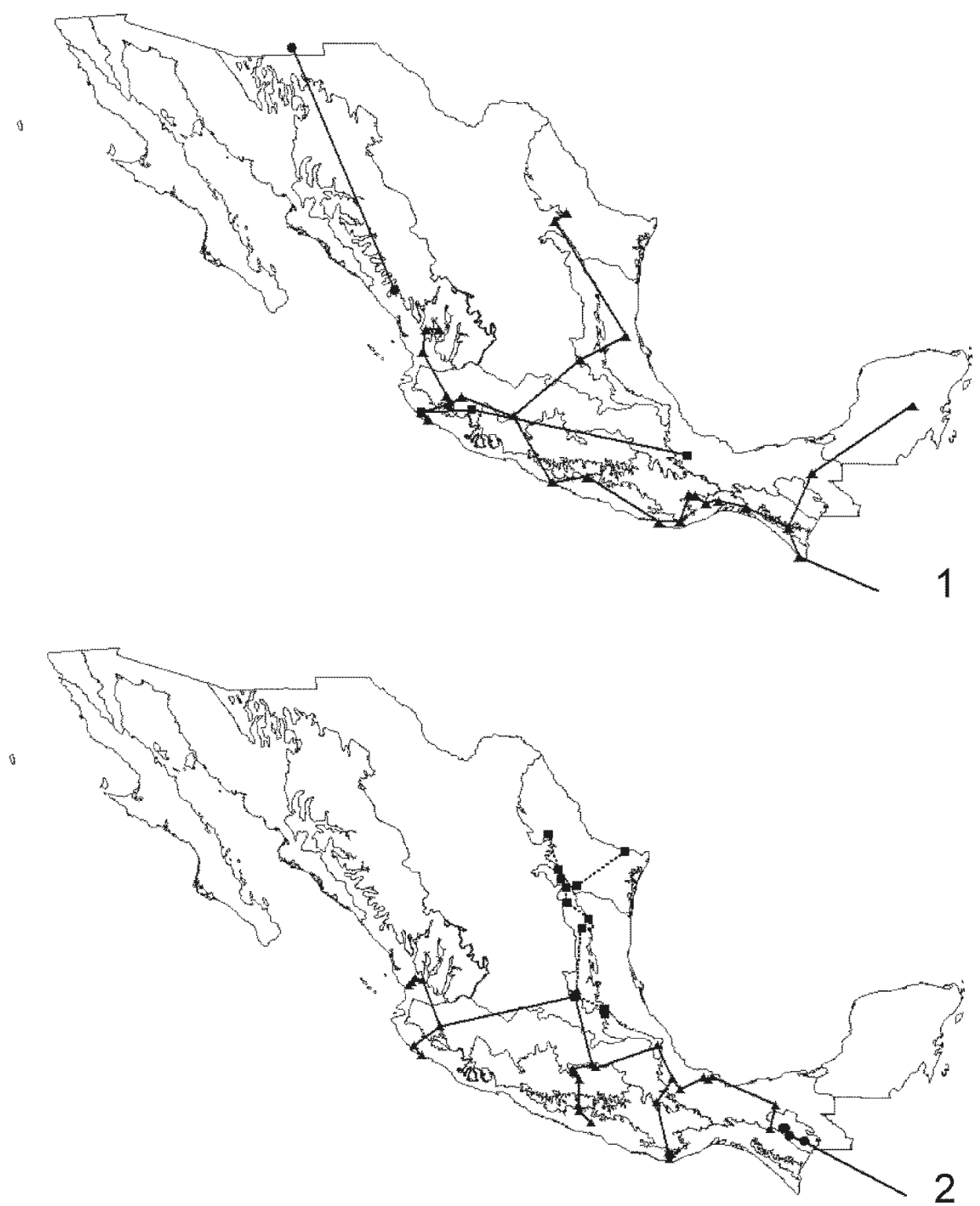

Figuras 1-2

Trazos individuales. 1, Pselliopus marmorosus (círculos), P. punctipes (triángulos) y P.flaviceps (cuadrados); 2, P. karlenae (círculos), P. nigropictus (triángulos) y P. latispina (cuadrados). 

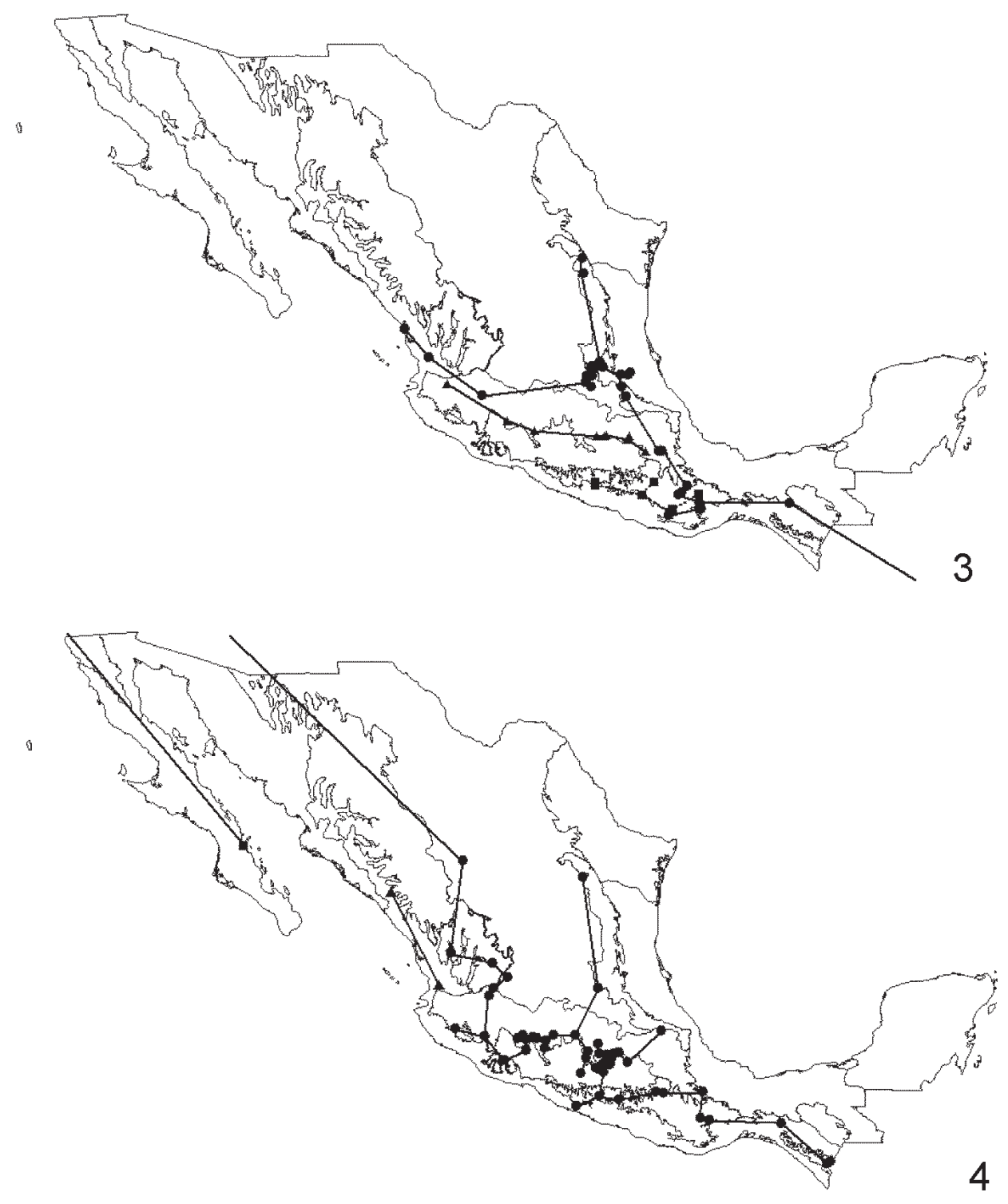

Figuras 3-4

Trazos individuales. 3, P. zebra (círculos), P. mexicanus (triángulos) y P. dantei (cuadrados); 4, P. inermis (círculos), P. lineaticeps (triángulos) y P. spinicollis (cuadro). 


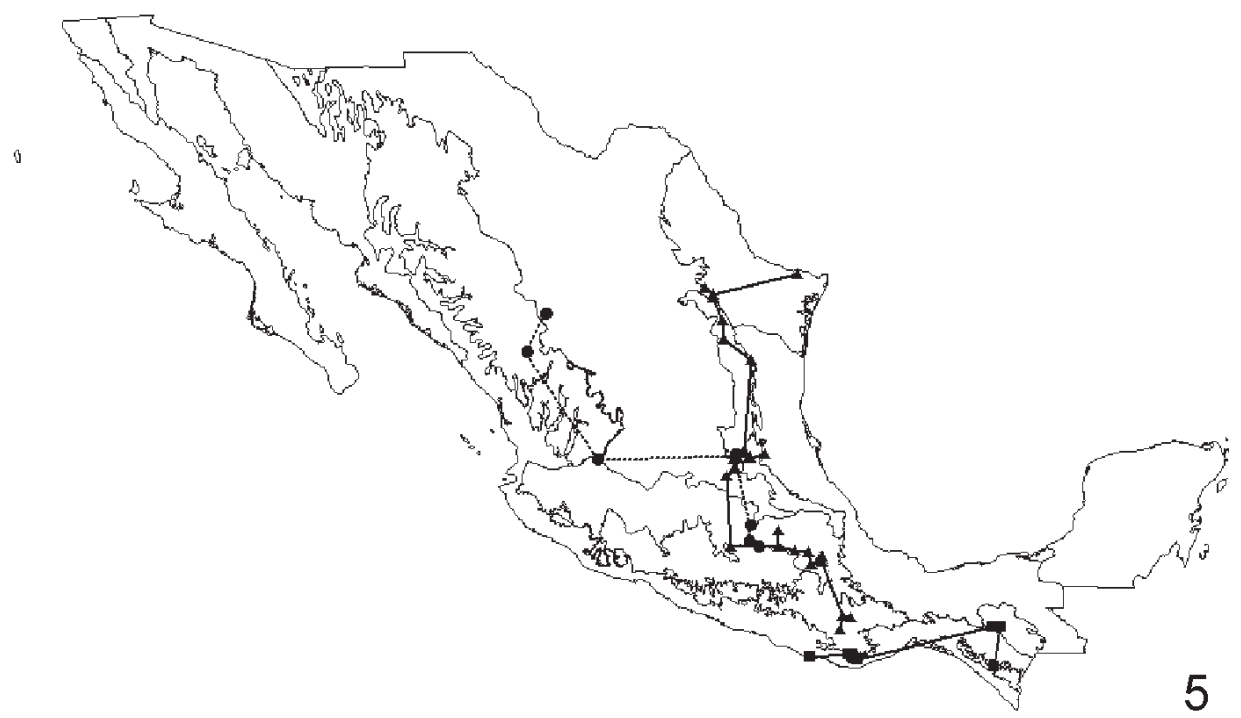

3

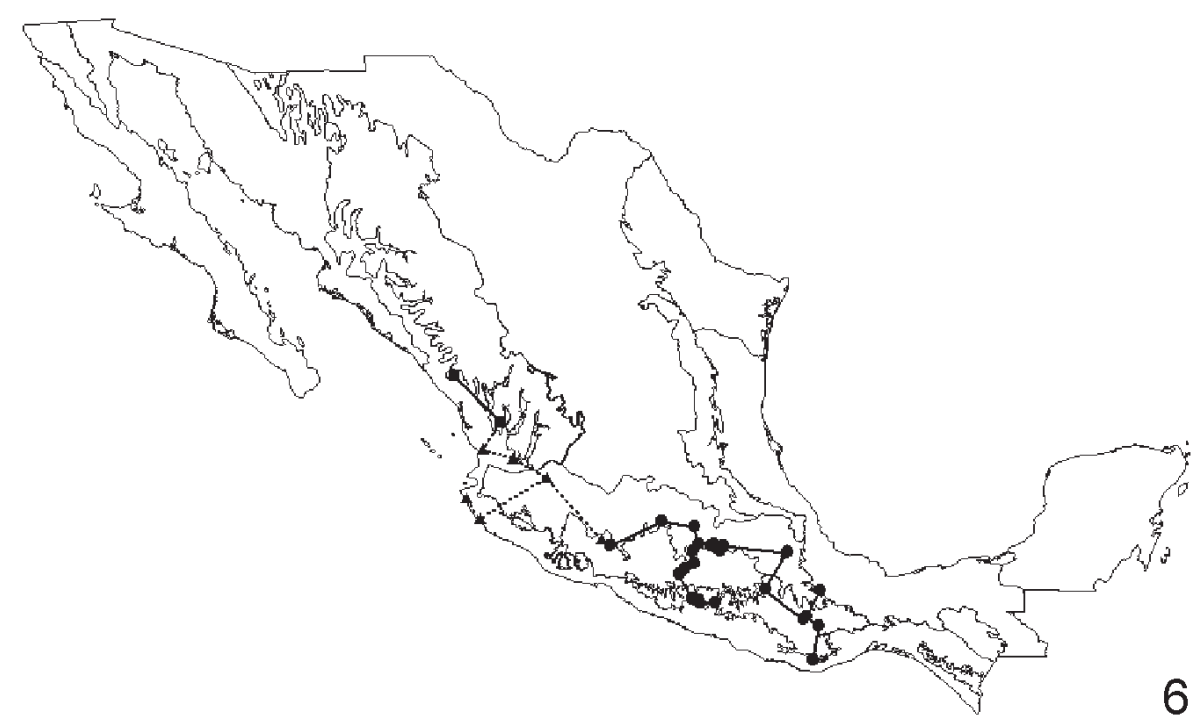

Figuras 5-6

Trazos individuales. 5, P. tuberculatus (círculos), P. cosmopolites (triángulos) y P. majesticus (cuadrados); 6, P. rufofasciatus (círculos), P. promeceops (triángulos) y P. mirabilis (cuadrados). 
de Chiapas y la Sierra Madre Occidental. Consideramos la sumatoria de ambos como un trazo generalizado (Fig. 9).

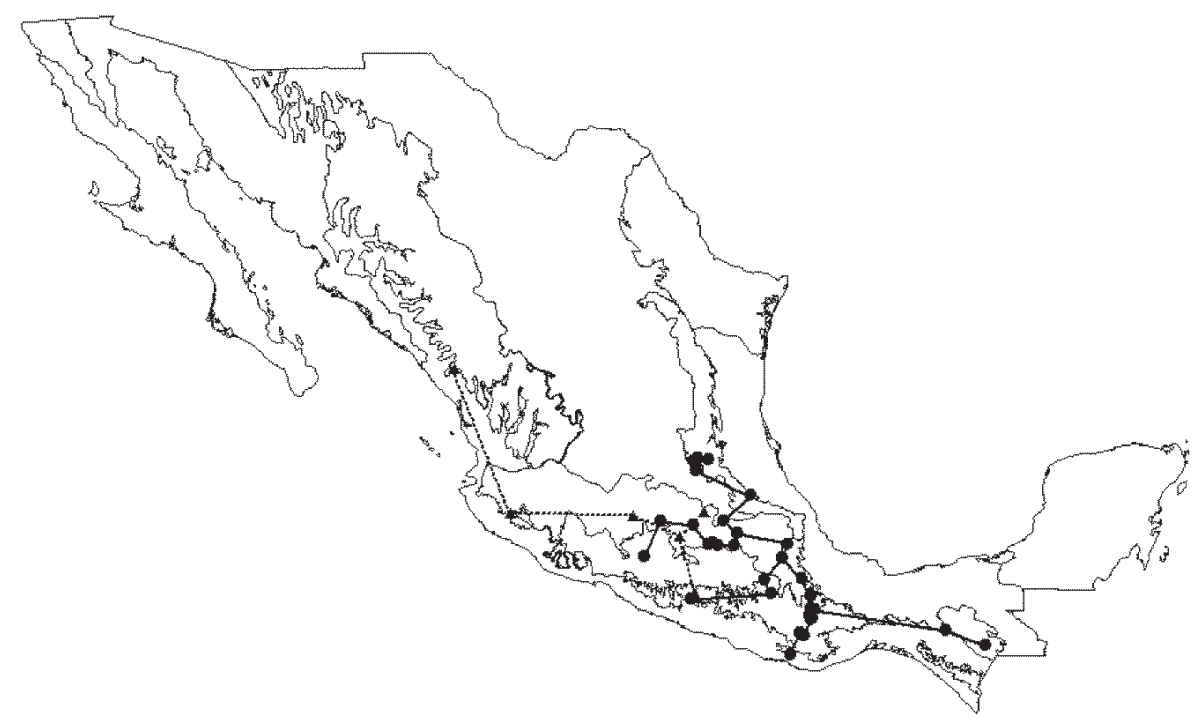

Figura 7

Trazos individuales. $P$. ventus (círculos) y P. ivanicus (triángulos).

\section{DISCUSIÓN}

Morrone y Márquez (2003) presentaron una aproximación preliminar a un atlas biogeográfico mexicano, con base en un análisis panbiogeográfico de la distribución de diferentes taxones de Coleoptera. En ella, determinaron cinco componentes bióticos principales: Neártico Californiano (provincias de California y Baja California), Neártico Continental (provincias de Sonora, Altiplano Mexicano y Tamaulipas), Mexicano de Montaña (provincias de la Sierra Madre Occidental, Sierra Madre Oriental, Eje Volcánico Transmexicano, Cuenca del Balsas y Sierra Madre del Sur), Antillano (provincia de la Península de Yucatán) y Mesoamericano (provincias del Golfo de México, Costa Pacífica Mexicana y Chiapas). Morrone (2005) asignó los dos primeros componentes bióticos a la región Neártica, el tercero a la Zona de Transición Mexicana (ZTM) y los dos últimos a la región Neotropical. Por consiguiente, la distribución de las especies de Pselliopus corresponde básicamente a los componentes Mexicanos de Montaña y Mesoamericano, es decir, a la región Neotropical y la Zona de Transición Mexicana.

Entre los taxones neotropicales que habitan la Zona de Transición Mexicana, Morrone (2005) y Huidobro et al. (2006) distinguieron dos elementos bióticos diferentes: el 

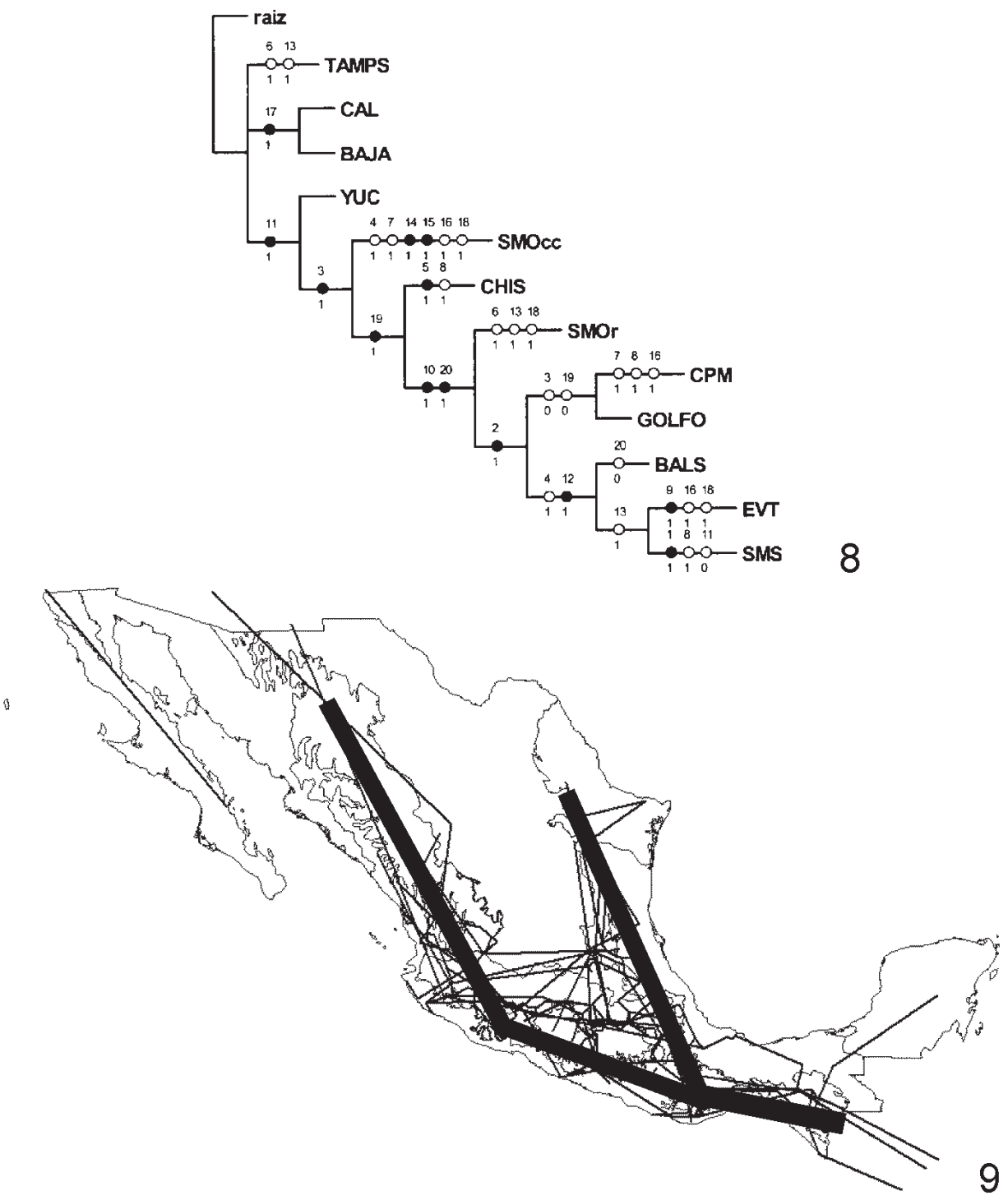

Figuras 8-9

8. Cladograma resultante del análisis de parsimonia de endemismos; 9, representación esquemática de los trazos generalizados obtenidos, con los trazos individuales superpuestos en segundo nivel. CAL, California; BAJA, Baja California; TAMPS, Tamaulipas; YUC, Península de Yucatán; SMOcc, Sierra Madre Occidental; SMOr, Sierra Madre Oriental; EVT, Eje Volcánico Transmexicano; BALS, Cuenca del Balsas; SMS, Sierra Madre del Sur; CPM, Costa del Pacífico Mexicano; GOLFO, Golfo de México; CHIS, Chiapas. 
Mesoamericano Tropical, que incluye taxones distribuidos por debajo de los $1000 \mathrm{~m}$, y el Mesoamericano de Montaña, que incluye taxones distribuidos por encima de los $1000 \mathrm{~m}$. En este caso, las especies de Pselliopus pertenecen al elemento Mesoamericano Tropical.

\section{Cuadro 3}

Matriz de datos para el análisis de parsimonia de endemismos. Filas: CAL, California; BAJA, Baja California; TAMPS, Tamaulipas; YUC, Península de Yucatán; SMOcc, Sierra Madre Occidental; SMOr, Sierra Madre Oriental; EVT, Eje Volcánico Transmexicano; BALS, Cuenca del Balsas; SMS, Sierra Madre del Sur; CPM, Costa del Pacífico Mexicano; GOLFO, Golfo de México; CHIS, Chiapas. Columnas: 1, Pselliopus dantei; 2, P. flaviceps; 3, P. inermis; 4, P. ivanicus; 5, P. karlenae; 6, P. latispina; 7, P. lineaticeps; 8, P. majesticus; 9, P. mexicanus; 10, P. nigropictus; 11, P. punctipes; 12 , P. rufofasciatus; 13 , P. cosmopolites; 14 , P. mirabilis; 15 , P. marmorosus; 16 , P. promeceops; 17, P. spinicollis; 18, P. tuberculatus; 19, P. ventus; 20, P. zebra.

\begin{tabular}{ll}
\hline raiz & 00000000000000000000 \\
CAL & 00000000000000001000 \\
BAJA & 00000000000000001000 \\
TAMPS & 00000100000010000000 \\
YUC & 00000000001000000000 \\
SMOcc & 00110010001001110100 \\
SMOr & 00100100011010000111 \\
EVT & 01110000111110010111 \\
BALS & 01110000011100000010 \\
SMS & 11110001010110000011 \\
CPM & 01000011011000010001 \\
GOLFO & 01000000011000000001 \\
CHIS & 00101001001000000010 \\
\hline
\end{tabular}

\section{AGRADECIMIENTOS}

A Roxana Acosta Gutiérrez por la ayuda en la realización de los trazos individuales. Especial agradecimiento a la Dirección General de Asuntos del Personal Académico (DGAPA) por el apoyo financiero al proyecto IN-223503-3.

\section{LITERATURACITADA}

Brailovsky, H. \& E. Barrera. 2004. Especies nuevas de Pselliopus (Hemiptera: Heteroptera: Reduviidae: Harpactorinae) de México. Anales del Instituto de Biología de la UNAM, Serie Zoología 75(1): 313-330.

Brailovsky, H., R. Mariño-Pérez \& E. Barrera. 2007. Cinco especies nuevas de Pselliopus Bergroth (Hemiptera: Heteroptera: Reduviidae: Harpactorinae: Harpactorini) para México. Revista Mexicana de Biodiversidad 78:85-98.

Craw, R. C., J. R. Grehan \& M. J. Heads. 1999. Panbiogeography: Tracking the history of life. Oxford Biogeography series 11, New York y Oxford. 
Croizat, L. 1958. Panbiogeography: Vols. 1 y 2. Publicado por el autor, Caracas. . 1964. Space, time, form: The biological synthesis. Publicado por el autor, Caracas.

Halffter, G. 1987. Biogeography of the montane entomofauna of Mexico and Central America. Annual Review of Entomology 32: 95-114.

Huidobro, L., J. J. Morrone, J. L. Villalobos \& F. Álvarez. 2006. Distributional patterns of freshwater taxa (fishes, crustaceans and plants) from the Mexican Transition Zone. Journal of Biogeography 33: 731-741.

Luna-Vega, I., O. Alcántara, J. J. Morrone \& D. Espinosa-Organista. 2000. Track analysis and conservation priorities in the cloud forests of Hidalgo, Mexico. Diversity and Distributions 6:137-143.

Maldonado-Capriles, J. 1990. Systematic catalogue of the Reduviidae of the World (Insecta: Heteroptera). Caribbean Journal of Science. Special Edition: 694 pp.

Mariño-Pérez, R. 2006. Revisión del género Pselliopus para México (Hemiptera: Heteroptera: Reduviidae: Harpactorinae). Tesis de Licenciatura, Facultad de Ciencias, UNAM, México, D.F. 58 pp.

Morrone, J. J. 2004. Homología biogeográfica: Las coordenadas espaciales de la vida. Cuadernos del Instituto de Biología 37, Instituto de Biología, UNAM, México, D.F. - 2005. Hacia una síntesis biogeográfica de México. Revista Mexicana de Biodiversidad 76: 207-252.

Morrone, J. J. \& J. V. Crisci. 1995. Historical biogeography: Introduction to methods. Annual Review of Ecology and Systematics 26: 373-401

Morrone, J. J. \& J. Márquez. 2003. Aproximación a un Atlas Biogeográfico Mexicano: componentes bióticos principales y provincias biogeográficas, pp. 217-220 en: Morrone, J. J. y J. Llorente (eds.), Introducción a la biogeografía en Latinoamérica: Conceptos, teorías, métodos y aplicaciones, Vol. 2, Las Prensas de Ciencias, UNAM, México, D.F.

Rosen, B. R. 1988. From fossils to earth history: Applied historical biogeography, pp. 437-481 en: Myers, A. A. y P. S. Giller (eds.), Analytical biogeography, Chapman and Hall, Londres.

Recibido: 9 de junio 2006

Aceptado: 2 de febrero 2007 